\title{
Suppression of the quantum-confined Stark effect in AlxGa1-xN/AlyGa1-yN corrugated quantum wells
}

\author{
A. A. Toropov, E. A. Shevchenko, T. V. Shubina, V. N. Jmerik, D. V. Nechaev, M. A. \\ Yagovkina, A. A. Sitnikova, S. V. Ivanov, Galia Pozina, J. P. Bergman and Bo Monemar
}

Linköping University Post Print

Tweet

N.B.: When citing this work, cite the original article.

Original Publication:

A. A. Toropov, E. A. Shevchenko, T. V. Shubina, V. N. Jmerik, D. V. Nechaev, M. A. Yagovkina, A. A. Sitnikova, S. V. Ivanov, Galia Pozina, J. P. Bergman and Bo Monemar, Suppression of the quantum-confined Stark effect in AlxGa1-xN/AlyGa1-yN corrugated quantum wells, 2013, Journal of Applied Physics, (114), 12.

http://dx.doi.org/10.1063/1.4822155

Copyright: American Institute of Physics (AIP) http://www.aip.org/

Postprint available at: Linköping University Electronic Press

http://urn.kb.se/resolve?urn=urn:nbn:se:liu:diva-100494 


\title{
Suppression of the quantum-confined Stark effect in $\mathrm{Al}_{x} \mathrm{Ga}_{1_{-x}} \mathrm{~N} / \mathrm{Al}_{y} \mathrm{Ga}_{1-y} \mathrm{~N}$ corrugated quantum wells
}

\author{
A. A. Toropov, ${ }^{1, a)}$ E. A. Shevchenko, ${ }^{1}$ T. V. Shubina, ${ }^{1}$ V. N. Jmerik, ${ }^{1}$ D. V. Nechaev, ${ }^{1}$ \\ M. A. Yagovkina, ${ }_{2}^{1}$ A. A. Sitnikova, ${ }^{1}$ S. V. Ivanov, ${ }^{1}$ G. Pozina, ${ }^{2}$ J. P. Bergman, ${ }^{2}$ \\ and B. Monemar ${ }^{2}$ \\ ${ }^{1}$ Ioffe Physical-Technical Institute, Russian Academy of Sciences, Polytekhnicheskaya 26, \\ 194021 St. Petersburg, Russia \\ ${ }^{2}$ Department of Physics, Chemistry and Biology, Linköping University, S-581 83 Linköping, Sweden
}

(Received 14 April 2013; accepted 10 August 2013; published online 25 September 2013)

\begin{abstract}
We report comparative studies of 6-nm-thick $\mathrm{Al}_{\mathrm{x}} \mathrm{Ga}_{1-\mathrm{x}} \mathrm{N} / \mathrm{Al}_{\mathrm{y}} \mathrm{Ga}_{1-\mathrm{y}} \mathrm{N}$ pyroelectric quantum wells (QWs) grown by plasma-assisted molecular beam epitaxy on c-sapphire substrates with a thick AlN buffer deposited under different growth conditions. The Al-rich growth conditions result in a 2D growth mode and formation of a planar QW, whereas the N-rich conditions lead to a 3D growth mode and formation of a QW corrugated on the size scale of 200-300 nm. Time-resolved photoluminescence (PL) measurements reveal a strong quantum-confined Stark effect in the planar QW, manifested by a long PL lifetime and a red shift of the PL line. In the corrugated QW, the emission line emerges $200 \mathrm{meV}$ higher in energy, the low-temperature PL lifetime is 40 times shorter, and the PL intensity is stronger ( $\sim 4$ times at $4.5 \mathrm{~K}$ and $\sim 60$ times at $300 \mathrm{~K})$. The improved emission properties are explained by suppression of the quantum-confined Stark effect due to the reduction of the built-in electric field within the QW planes, which are not normal to the [0001] direction, enhanced carrier localization, and improved efficiency of light extraction. ( 2013 AIP Publishing LLC. [http://dx.doi.org/10.1063/1.4822155]
\end{abstract}

\section{INTRODUCTION}

Heterostructures based on AlGaN solid alloys are currently used for ultraviolet (UV) light-emitting diodes (LEDs), which are crucial to many upcoming applications such as air/water purification, detection of different substances, and light-sensitive technologies in medicine. However the external quantum efficiency of the currently produced deep UV AlGaN LEDs with the emission wavelength shorter than $275 \mathrm{~nm}$ remains very low at $1 \%-2 \%,{ }^{1-3}$ as compared to over $70 \%$ efficiencies of blue GaN/InGaN LEDs. The most important reasons for this are degradation of both internal quantum efficiency and light-extraction efficiency in the employed $\mathrm{Al}_{\mathrm{x}} \mathrm{Ga}_{1-\mathrm{x}} \mathrm{N} / \mathrm{Al}_{\mathrm{y}} \mathrm{Ga}_{1-\mathrm{y}} \mathrm{N}$ quantum wells (QWs) with high aluminum content.

The internal quantum efficiency suffers from a high density of threading dislocations generated in the buffer layers and from the quantum confined Stark effect (QCSE) induced by strong spontaneous polarization in Al-rich solid alloys grown on c-plane sapphire. ${ }^{4}$ Another disadvantage of the Alrich $\mathrm{AlGaN}$ heterostructures is the phenomenon of switching the polarization of the emitted light from transverse electric (TE) polarization to transverse magnetic (TM) polarization as the $\mathrm{Al}$ content increases. ${ }^{5}$ The origin of this effect is the crossover of heavy-hole and split-off-hole bands in AlGaN at a certain $\mathrm{Al}$ composition. This circumstance defines an essential drop of the light-extraction efficiency in topemitting c-plane UV LEDs because light propagating along the c-axis must have TE polarization. For the structures

\footnotetext{
a) Author to whom correspondence should be addressed. Electronic mail: toropov@beam.ioffe.ru
}

grown on c-sapphire, the wavelength of crossover is at approximately $300 \mathrm{~nm} .{ }^{5} \mathrm{In}$ view of these problems, thorough design of the buffer, as well as of the whole heterostructure, is of particular importance.

Recently, close attention was focused on the growth of AlGaN QW structures along nonpolar and semipolar directions with diminished polarization-related electric fields inside the QWs. ${ }^{6,7}$ However the non-c-plane heterostructures still contain an enhanced density of nonradiative recombination centers that prevents their applications to efficient LEDs. In the polar c-plane AlGaN QWs the QCSE reduces the optical transition probability due to the polarization of electrons and holes confined in the QW. This results in an enhancement of the radiative lifetime and, hence, in a reduction of the internal quantum efficiency. The emission efficiency can be enhanced by decreasing the QW width below $\sim 3 \mathrm{~nm}$ that partly suppresses the QCSE. ${ }^{4}$ On the other hand the possibility of using thicker QWs with deeper electron and hole levels could be especially advantageous in order to overcome the effect of efficiency droop in high-power LEDs.

It was previously shown that the QCSE in a GaN/ $\mathrm{Al}_{0.2} \mathrm{Ga}_{0.8} \mathrm{~N} \mathrm{QW}$ can be largely suppressed by the growth on randomly textured $\mathrm{GaN}$ templates. ${ }^{8}$ In this paper, we use a similar technique to improve luminescence efficiency of $\mathrm{Al}_{\mathrm{x}} \mathrm{Ga}_{1-\mathrm{x}} \mathrm{N} / \mathrm{Al}_{\mathrm{y}} \mathrm{Ga}_{1-\mathrm{y}} \mathrm{N}$ QWs grown on c-sapphire, emitting near $300 \mathrm{~nm}$. We demonstrate that the strength of the QCSE in such QWs can be controlled by varying growth conditions and, hence, morphology of a thick AlN buffer deposited on a c-sapphire substrate. By means of transmission electron microscopy (TEM), cathodoluminescence (CL) spectroscopy, and time-resolved (TR) photoluminescence (PL) 
spectroscopy we show that N-rich conditions during the growth of the AlN buffer result in the formation of a corrugated QW with the strongly suppressed QCSE and enhanced emission efficiency as compared to an identical reference QW structure grown with a planar buffer. In addition, it was found that the length-scale of the obtained corrugation is of the order of the emitting wavelength, which should facilitate the efficiency of light extraction.

\section{SAMPLES AND EXPERIMENTAL TECHNIQUES}

In this study we compare properties of two samples (A and $\mathrm{B}$ ) with the same general design, grown by plasmaassisted molecular beam epitaxy (PA MBE) on c-sapphire substrates using different AlN buffer layers. In both samples the growth of the $2-\mu$ m-thick AlN layers was initiated at a low substrate temperature $\left(T_{S} \sim 550^{\circ} \mathrm{C}\right)$ under N-rich conditions with the group-III-to-nitrogen flux ratio $\left(\mathrm{F}_{\mathrm{III}} / \mathrm{F}_{\mathrm{N}}\right)$ being about 0.6, which led to a three-dimensional (3D) growth mode. After $130 \mathrm{~nm}$ of growth, the substrate temperature in both samples was raised up to $T_{S} \sim 800{ }^{\circ} \mathrm{C}$. In sample $\mathrm{A}$, the $3 \mathrm{D}$ growth mode was switched to the flat two-dimensional (2D) one by increasing the $\mathrm{Al}$ flux to ensure metal ( $\mathrm{Al}$ ) rich conditions with $\mathrm{F}_{\mathrm{III}} / \mathrm{F}_{\mathrm{N}} \sim 1.1-1.2$. In sample $\mathrm{B}$, the flux ratio was kept constant $\left(F_{I I I} / F_{N} \sim 0.6\right)$ during growth of the whole AlN buffer layer. In both structures, the buffer was followed by $500-n m$-thick $\mathrm{Al}_{\mathrm{x}} \mathrm{Ga}_{1-\mathrm{x}} \mathrm{N}(\mathrm{x} \sim 0.45 \pm 0.03)$ layers grown at a relatively low temperature $T_{S} \sim 700{ }^{\circ} \mathrm{C}$ and metal-rich conditions with similar values of metal and activated nitrogen fluxes. The flux ratio $F_{I I I} / F_{N} \sim 1.6$ corresponding to the transition from $3 \mathrm{D}$ to $2 \mathrm{D}$ growth mode was used for both samples. ${ }^{9,10}$ Both samples contain an AlGaN QW with the nominal thickness of $6 \mathrm{~nm}$ and the aluminum content by 10 percent less than that in the barrier layer. The QW was grown by a submonolayer digital alloying technique providing high accuracy of composition control in the AlGaN QW with respect to that in the barrier. ${ }^{9}$ The thickness of the capping AlGaN barrier was $75 \mathrm{~nm}$. Thus, the structures mainly differ in the growth regime of the AIN buffer. Surface morphology and growth rate of all layers were controlled in situ by using both reflected high energy electron diffraction (RHEED) and optical reflectometry.
High-resolution X-ray diffraction (HRXRD) measurements were performed on a D8Discover (Bruker AXS) set up with rotated anode using $\mathrm{Cu} K \alpha$ radiation. Reciprocal space maps (RSM) were obtained with a four-bounce Ge [220] monochromator and a PSD detector LynxEye. The intrinsic crystal morphology of the samples was explored by taking cross-sectional transmission electron microscopy (TEM) images and room-temperature plan-view images of spatially resolved CL and scanning electron microscopy (SEM). Low-temperature PL spectra were measured under the conditions of $\mathrm{cw}$ excitation by emission of a Xe lamp, passed through a monochromator. The spectra of timeresolved PL were measured using a triple-frequency femtosecond Ti-sapphire laser (Coherent Mira 900) and a Hamamatsu streak camera. The width of the laser pulse was $\sim 150 \mathrm{fs}$, the repetition rate was $76 \mathrm{MHz}$, and the typical average excitation power density was in the range $0.5-1.0 \mathrm{~W} / \mathrm{cm}^{2}$. The excitation wavelength was $260 \mathrm{~nm}$, and the total time resolution of the PL set-up was estimated as $\sim 15 \mathrm{ps}$.

\section{RESULTS AND DISCUSSION}

\section{A. Structural studies and cathodoluminescence}

Monitoring of the RHEED patterns revealed a striking difference between the samples: a streaky pattern was observed during the growth of the high-temperature AIN buffer layers and the AlGaN layers in sample A, while a bright spotty pattern remained practically unchanged during the whole growth of sample B. Figure 1 shows the RHEED patterns as well as surface SEM and cross-sectional TEM images taken in samples A (Figs. 1(a) and 1(c)) and B (Figs. $1(\mathrm{~b})$ and $1(\mathrm{~d})$ ), which evidence either $2 \mathrm{D}$ (sample A) or 3D (sample B) surface morphologies. These results confirm that the metal-rich stoichiometric conditions, different for the AlN and AlGaN layers, enhance surface mobility of adatoms, leading to a $2 \mathrm{D}$ growth mode. ${ }^{9}$ One should note that the morphology of sample B remains 3D during the growth of the AlGaN layers despite the metal-rich conditions used, which can be related to still insufficient metal-enrichment $\left(F_{I I I} / F_{N} \sim 1.6\right)$ to flatten the AlGaN surface for the relatively small thickness of the layers $(<1 \mu \mathrm{m})$. The TEM images
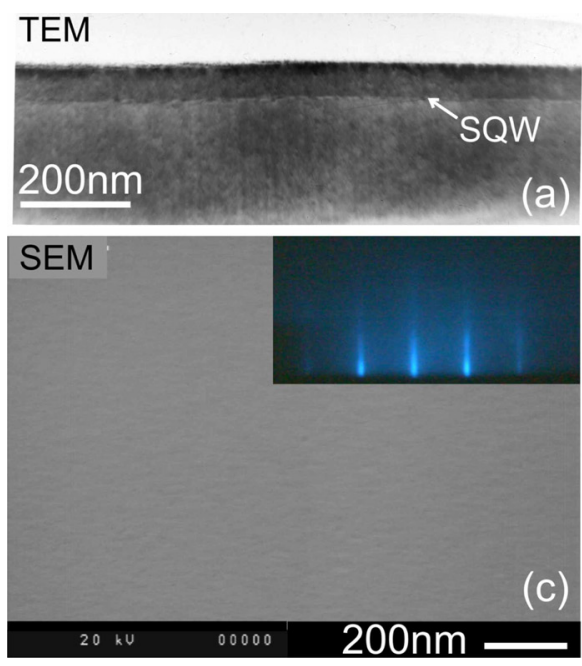
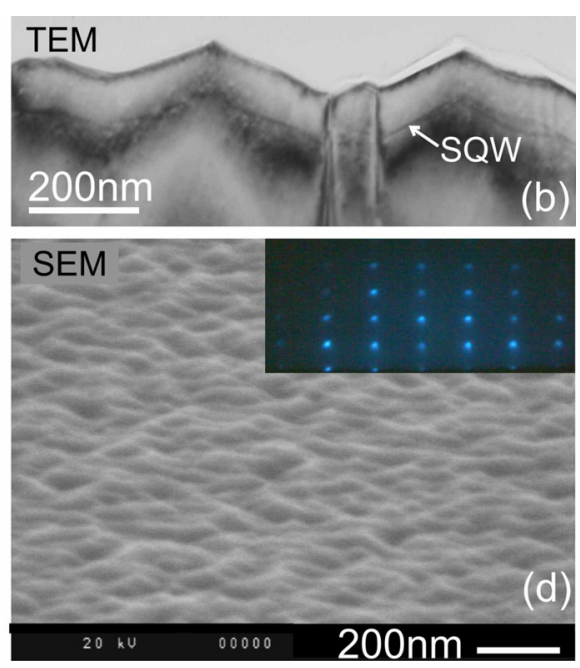

FIG. 1. Cross-sectional TEM images of samples A (a) and B (b). Surface SEM images of samples A (c) and B (d) with insets illustrating corresponding RHEED patterns. 

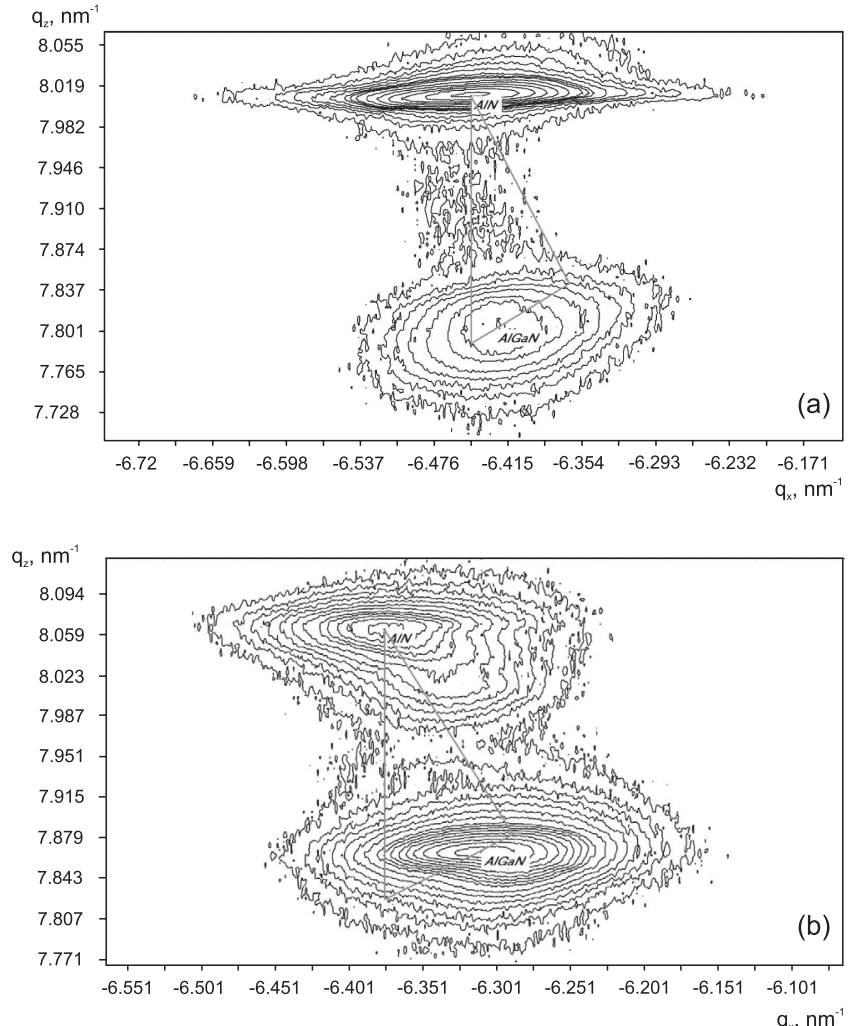

FIG. 2. Reciprocal space maps for the asymmetric (ĪI24) reflections of samples A (a) and B (b).

exhibit the flat QW in the planar structure (sample A), while in sample B it is corrugated reflecting the $3 \mathrm{D}$ wavy morphology of the underlying layers.

Figure 2 shows the RSM's of the studied AlGaN/AlN $\mathrm{QW}$ heterostructures. Analysis of these maps reveals $\mathrm{Al}$ contents close to $\mathrm{x} \sim 0.45$ in the ternary barrier layers of both samples. However, these samples exhibit big difference in the degree of relaxation of the AlGaN layers $R=1-\left(\varepsilon_{\text {meas }} /\right.$ $\left.\varepsilon_{c o h}\right)$, where $\varepsilon_{\text {meas }}$ is the elastic strain value determined from the RSM analysis and $\varepsilon_{c o h}$ is the elastic strain in the fully coherent epitaxial layer. ${ }^{11}$ Modeling in accordance with elastic strain theory revealed a relatively low degree of relaxation for sample A with the 2D morphology $(R=0.25)$, while sample $B$ with the 3D morphology suffers from stronger strain relaxation $(\mathrm{R}=0.75)$.

For both samples, the average density of screw threading dislocations in the neighborhood of the QW is in the range $(5-6) \times 10^{9} \mathrm{~cm}^{-2}$, while the density of edge and mixed dislocations reaches $(1-3) \times 10^{10} \mathrm{~cm}^{-2}$ as counted from TEM images taken at $\mathrm{g}=\{0002\}$ and $\mathrm{g}=\{01-10\}$ two-beam imaging conditions, respectively. ${ }^{11}$ It should be noted that in sample A the dislocations are distributed homogeneously, while in sample B they tend to bunch beneath the dips of the topographic relief rather than beneath the protrusions (see Fig. 1(b)).

This tendency is confirmed by plane-view images of spatially resolved CL (Fig. 3(a)) and SEM (Fig. 3(b)) measured at the same place of sample B. The CL wavelength corresponds to the peak of the $\mathrm{QW}$ emission. There is clear correlation between the CL and SEM images, confirming CL
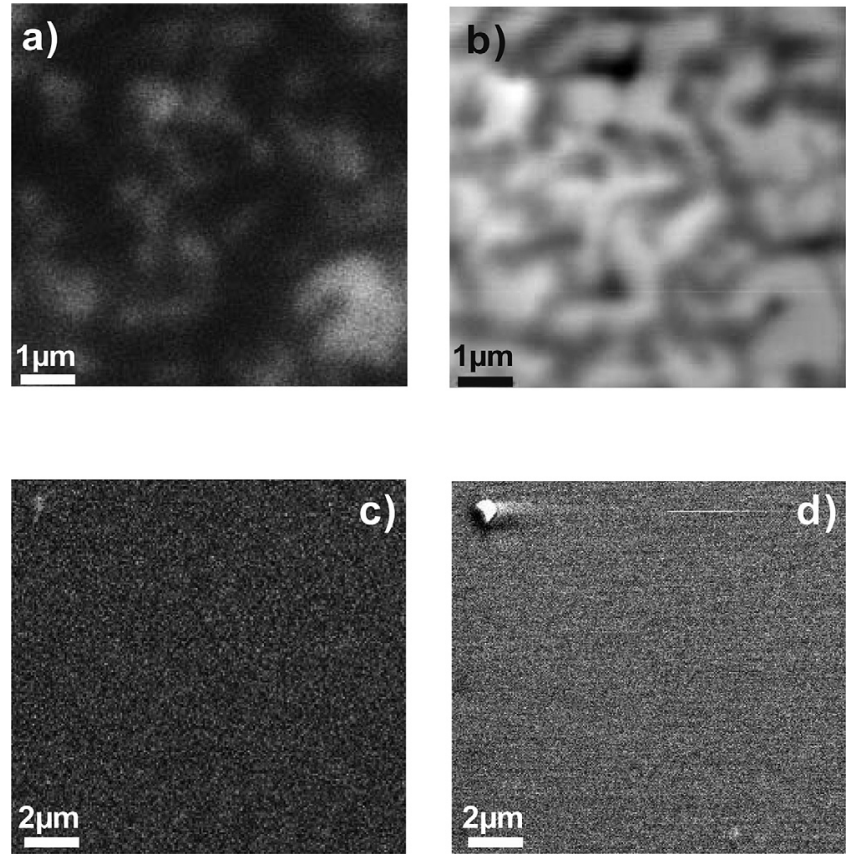

FIG. 3. Plan-view CL (a), (c) and SEM (b), (d) images of the same place in samples B (a), (b) and A (c), (d).

enhancement when the electron-hole pairs are excited by the electron beam within protrusions (bright areas in the SEM image in Fig. 3(b)), where the dislocation density is reduced. One should note that the corresponding CL images measured in sample A are fairly uniform and featureless (Figs. 3(c) and 3(d)). To explain this observation we speculate that at the measurement temperature $300 \mathrm{~K}$ the spreading of excited carriers in this sample is larger than the average distance between the dislocations.

\section{B. Continuous-wave and time-resolved photoluminescence}

Figure 4(a) represents low-temperature PL spectra measured in both samples under the conditions of $c w$ excitation. The spectra exhibit emission lines originating both from the QW and the barriers. In sample A, these two lines are relatively narrow and well separated, whereas in sample B the respective peaks are wider and merge together. The intensity at the QW emission line maximum in sample B at $20 \mathrm{~K}$ is nearly 4 times higher than in sample A. Besides, this peak in sample B is shifted toward higher energies by about $200 \mathrm{meV}$. Figure 4(b) shows the time-integrated PL spectra measured under pulsed excitation conditions at $300 \mathrm{~K}$. At this temperature, the PL from the barriers is negligibly weak, whereas the integral QW PL intensity in sample B is about 60 times larger than in sample A.

The spectrally and time-integrated intensity of the QW PL line is plotted in Fig. 5 versus temperature. Below the temperatures about $120-140 \mathrm{~K}$, the intensity decrease is relatively weak and similar in both samples. Above this temperature, the PL intensity in sample A falls much faster, being at $300 \mathrm{~K}$ about 140 times weaker than at $4.5 \mathrm{~K}$. In sample B, the respective PL reduction is only seven-fold.

Figure 6 represents PL decay curves measured at the peaks of the QW emission in both A and B samples. At low 


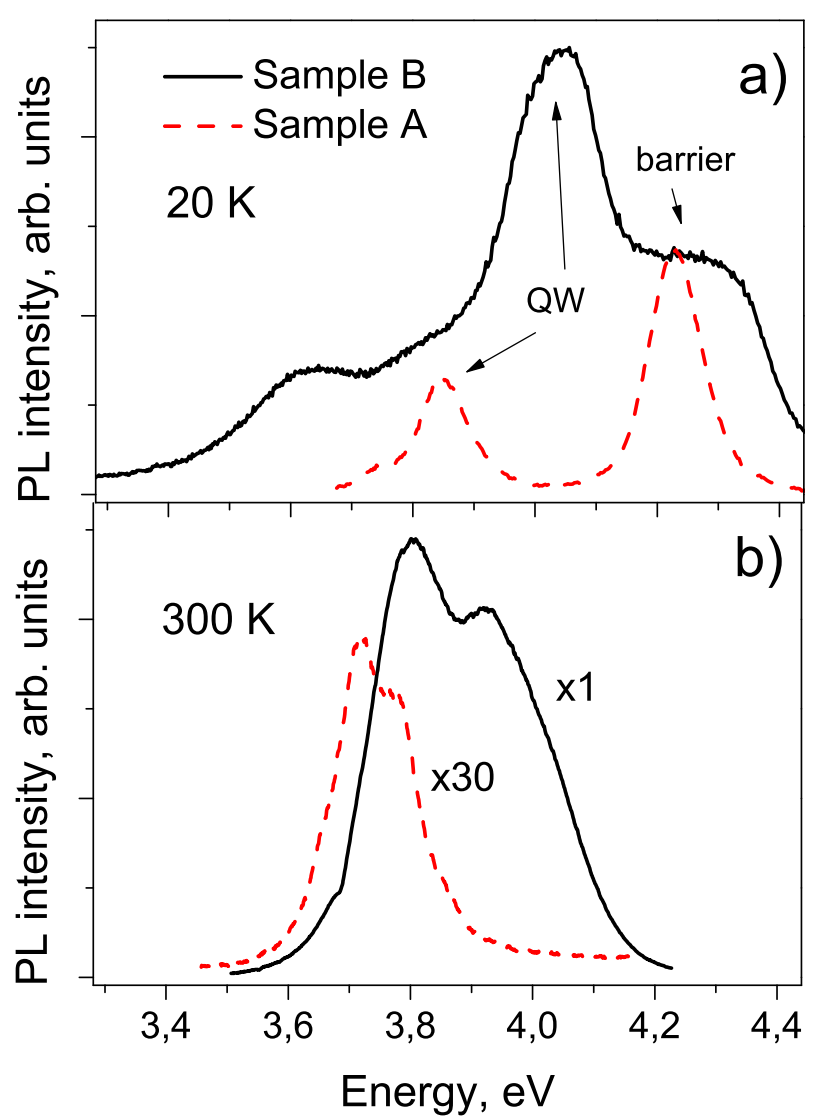

FIG. 4. PL spectra measured in samples A and B under $c w$ excitation at $20 \mathrm{~K}$ (a) and under pulsed excitation at $300 \mathrm{~K}$ (b).

temperatures below $\sim 140 \mathrm{~K}$, the PL decay in sample $\mathrm{A}$ is rather long. One can see in Fig. 6(a) that a significant part of the PL signal does not decay during the period between the laser pulses $(\sim 13 \mathrm{~ns})$. While precise determination of the decay parameters is hampered by the relatively high repetition rate of the laser pulses, one can be sure that the respective decay time constant is in the range of tens of nanoseconds.

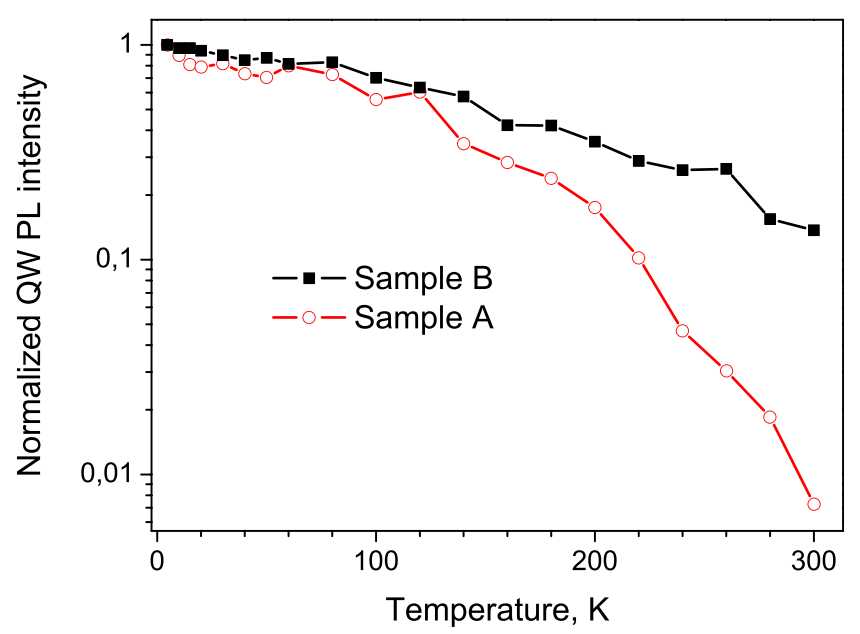

FIG. 5. Time- and spectrally integrated QW PL intensity versus temperature in sample A (empty circles) and B (filled squares). The curves are normalized to the intensity obtained at the lowest temperature $4.5 \mathrm{~K}$.

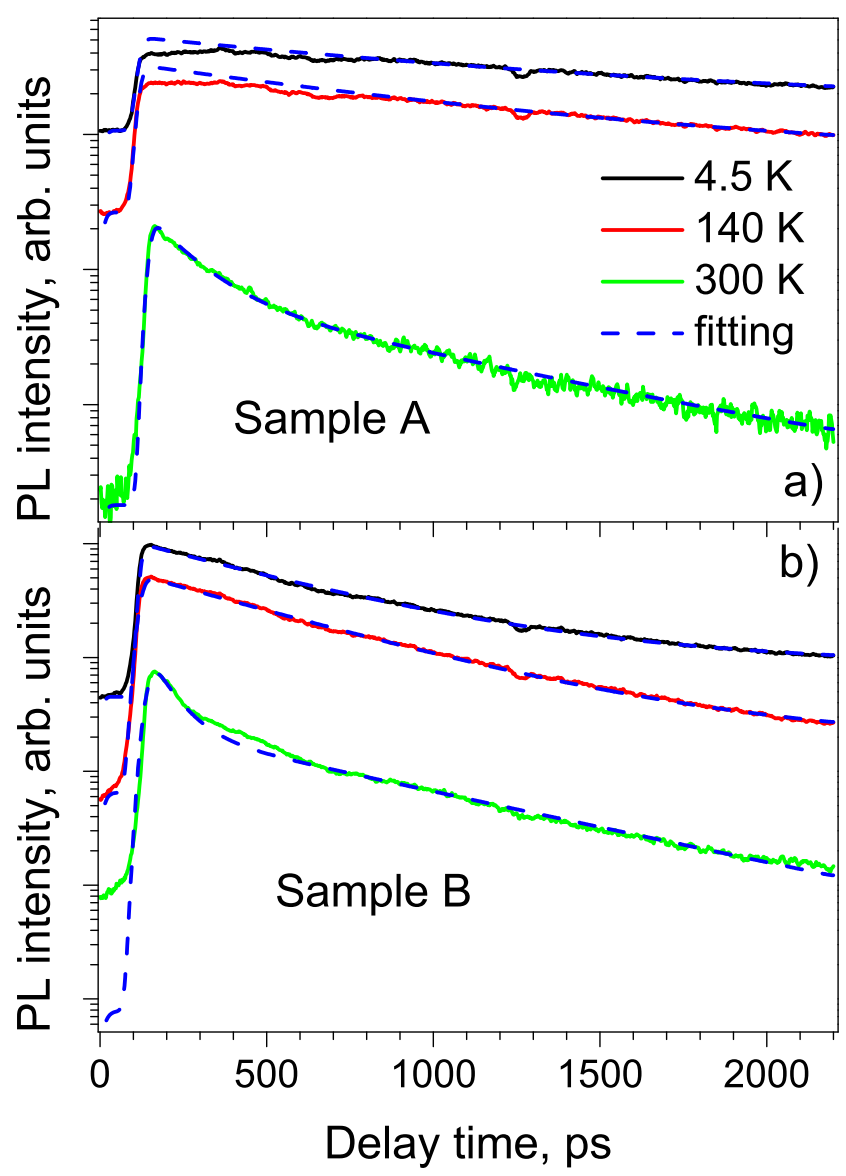

FIG. 6. Decay curves measured at the QW PL maximum in sample A (a) and B (b) at three selected temperatures: $4.5 \mathrm{~K}, 140 \mathrm{~K}$, and $300 \mathrm{~K}$.

Dashed lines in Fig. 6 show the results of fitting performed in the model of two-exponential decay, taking into account contributions from multiple laser pulses. According to this simulation nearly $30 \%$ of the population of photoexcited carriers decays at $4.5 \mathrm{~K}$ with the characteristic decay constant as large as $\sim 20 \mathrm{~ns}$. We believe that this decay constant represents at low temperature the radiative lifetime of localized excitons, which are responsible for the QW emission. When the temperature increases above $\sim 140 \mathrm{~K}$, the PL decay gradually becomes faster, and at $300 \mathrm{~K}$ the dominant decay time constant is as low as about 130 ps. Note that at all temperatures the decay curves cannot be fitted by a single decaying exponent and even two-exponential fitting is at some temperatures not satisfactory. In sample B the PL decay is much faster; at low temperatures most of the signal decays within one period of the excitation pulses with the dominant characteristic decay constant about 500 ps.

One can see in Fig. 6 that the decrease of the timeintegrated PL intensity with temperature can be separated into two contributions. First, the temperature rise causes an increase of the non-radiative recombination rate $\Gamma_{n r}=\frac{1}{\tau_{n r}}$ and, hence, the reduction of the internal quantum yield $Y=\frac{\Gamma_{r}}{\Gamma_{r}+\Gamma_{n r}}$, where $\Gamma_{r}=\frac{1}{\tau_{r}}$ is the radiative recombination rate and $\tau_{n r}$ and $\tau_{r}$ are, respectively, the non-radiative and radiative decay time constants. Besides, the increase in temperature results in a certain quenching of the PL intensity immediately after excitation, which can be attributed to 
temperature variation of the transport efficiency of photoexcited carriers through the thick AlGaN cap layer. This interpretation correlates with the quenching behavior of the time-integrated PL shown by Fig. 5. Below $\sim 140 \mathrm{~K} \Gamma_{n r}$ is much smaller than $\Gamma_{r}$ for both samples, and the PL quenching is governed by the same mechanism connected to carrier losses during the transport through the AlGaN barrier. At higher temperatures, $\Gamma_{n r}$ becomes comparable to $\Gamma_{r}$ (or less) that leads to the decrease in the internal quantum yield. The important factor defining faster quenching of the timeintegrated PL in sample A is the longer radiative lifetime.

\section{The quantum confined Stark effect and exciton localization}

The observed long lifetime of photoexcited carriers in sample A can be explained by simultaneous action of two factors, namely, the QCSE in a relatively wide QW, and localization of carriers preventing their diffusion toward nonradiative recombination centers, at least at low temperatures. To estimate the radiative lifetime of excitons in the pyroelectric QW we adopt a variational approach with the trial envelope wave function of the ground s-like exciton state expressed as ${ }^{12,13}$

$$
\begin{aligned}
\Psi_{e x}\left(\rho, z_{e}, z_{h}\right)= & \frac{1}{\sqrt{N}} \varphi_{e}\left(z_{e}\right) \varphi_{h}\left(z_{h}\right) \\
& \times \exp \left(-\sqrt{\rho^{2}+\sigma^{2}\left(z_{e}-z_{h}\right)^{2}} / \lambda\right) .
\end{aligned}
$$

Here $z$ is the coordinate along the growth axis, $z_{e}\left(z_{h}\right)$ is the $z$ coordinate of the electron (hole), $\rho=$ $\sqrt{\left(x_{e}-x_{h}\right)^{2}+\left(y_{e}-y_{h}\right)^{2}}$ is the coordinate of the relative motion of the electron and hole, $\varphi_{e}$ and $\varphi_{h}$ are singleparticle wave functions formed by linear combinations of Airy functions, and $N$ is a normalizing constant. Equation (1) includes two variational parameters, $\lambda$ and $\sigma ; \lambda$ corresponds to the exciton Bohr radius and $\sigma$ is a measure of the exciton dimensionality. The radiative lifetime of the exciton is calculated as

$$
\tau=\frac{1}{\pi a_{B}^{3} k_{0} \omega_{L T}|\Phi(0)|^{2}},
$$

where $a_{B}$ and $\omega_{L T}$ are the exciton Bohr radius and longitudinal-transverse exciton splitting in the bulk QW material, $k_{0}$ is the wave vector of the emitted photon, and the electron-hole overlap $\Phi(0)$ is given by

$$
\Phi(0)=\int d q \Psi\left(\rho=0, z_{e}=q, z_{h}=q\right) .
$$

Material parameters in the calculation were taken from Refs. 14-16. The electric field was taken as homogeneous both in the well and barriers.

The calculation is complicated by the poor knowledge of many band parameters of AlGaN ternary alloys with high content of $\mathrm{Al}$, such as the deformation potentials and the band-gap bowing factor. ${ }^{14}$ Therefore, we preferred to estimate the QW band line-ups from the experimental lowtemperature PL spectra. In particular, the band gap energy $E_{g, b}$ of the $\mathrm{Al}_{\mathrm{y}} \mathrm{Ga}_{1-\mathrm{y}} \mathrm{N}$ barriers was estimated from the exciton resonant energy as $E_{e x}=E_{P L}+0.6 \Delta E_{P L}$, where $E_{P L}$ is the energy of the PL peak and $\Delta E_{P L}$ is its full width at half maximum. ${ }^{17}$ Then the band gap energy is calculated by adding the exciton binding energy obtained by linear interpolation between the known values for $\mathrm{GaN}$ and $\mathrm{AlN}$ for $y=0.45$ that is the technologically intended composition. Furthermore, the difference in the band gap energy between the QW and the barriers, corresponding to the $10 \%$ difference in the compositions, was taken constant, since this quantity only weakly depends on the strain and composition variations. A small arbitrary value $10^{3} \mathrm{~V} / \mathrm{cm}$ was used in this calculation as the barrier electric field, since we assume that the polarization-induced electric field in the thick barriers is much weaker than in the thin QW. ${ }^{4}$ Actually, it was found that the exciton parameters only weakly depend on the barrier electric field in a wide range between zero and $10^{4} \mathrm{~V} / \mathrm{cm}$.

According to the performed calculations, the exciton energy matching the position of the experimentally observed PL peak is obtained provided that the QW intrinsic electric field is as high as $7 \times 10^{5} \mathrm{~V} / \mathrm{cm}$. One should note that the estimation of the intrinsic electric field in this pyroelectric structure, performed in the simplest model of an undoped $\mathrm{QW},{ }^{4}$ provides a comparable value $7.5 \times 10^{5} \mathrm{~V} / \mathrm{cm}$. The respective calculated radiative lifetime of excitons was $15 \mathrm{~ns}$. Hence, we conclude that both emission photon energy and lifetime of the QW PL in sample A is governed by the strong QCSE induced by the spontaneous and piezoelectric polarizations, in perfect agreement with the structure design.

In spite of the same nominal thickness of the QW and the same intended $\mathrm{AlGaN}$ compositions in samples A and B, the PL lifetime in the latter sample is shorter, the PL intensity is larger, and the PL line position is shifted toward higher energies. All these observations are consistent with a considerable damping of the QCSE in this structure. Indeed, the energy of the QW PL line in sample B corresponds to the QW electric field $\sim 2.6 \times 10^{5} \mathrm{~V} / \mathrm{cm}$ and to the exciton radiative lifetimes $\sim 100 \mathrm{ps}$. This decay time constant is smaller than the experimentally obtained value $\sim 500 \mathrm{ps}$. One should note nevertheless that the PL from barriers in both samples demonstrates very similar decay time, which implies that the dominant effect defining the decay of the QW PL in this sample is exciton localization by potential fluctuations rather than the QCSE in an ideal plane QW. ${ }^{18,19}$ Following Ref. 8 we tentatively attribute the observed reduction of the intrinsic electric field in the corrugated QW of sample B to deviation of the normal to the QW planes from the [0001] direction of the polarization vector. The respective suppression of the QCSE should be favorable for the internal quantum yield, especially at elevated temperatures facilitating nonradiative recombination.

One more specific feature of sample B is broadening of the PL peaks as well as emergence of a tail of emission and additional PL lines at lower energies (see Fig. 4). It could be explained by enhanced compositional inhomogeneity in both barrier and QW layers. Such inhomogeneities in the 
$\mathrm{Al}_{\mathrm{x}} \mathrm{Ga}_{1-\mathrm{x}} \mathrm{N}$ layers with the middle-range $\mathrm{Al}$ content $(0.25<\mathrm{x}<0.5)$, grown by PA MBE, can originate from a considerable difference in surface mobility of $\mathrm{Ga}$ and $\mathrm{Al}$ adatoms in the middle growth-temperature range $\left(700-750^{\circ} \mathrm{C}\right) .^{20-22}$ However, the reduction of the growth temperature below $700{ }^{\circ} \mathrm{C}$ and maintaining the slightly metal-rich growth conditions, realized for the $\mathrm{AlGaN}$ growth in both A and B samples, should yield homogeneous alloys, as it occurred in the case of sample A. Thus, one can conclude that much stronger alloy inhomogeneity in sample B is generally induced by the 3D surface morphology inherited from the AlN buffer layer. The strong carrier localization caused by the enhanced compositional fluctuations is also advantageous for increasing the intrinsic quantum yield since it prevents the carriers from moving to the nonradiative recombination centers. ${ }^{3}$

In addition to the suppression of the QCSE and the enhanced carrier localization, the PL enhancement in the corrugated QW can be partly attributed to the increase of light extraction efficiency. Indeed, only less than $4 \%$ of the emitted light can be extracted from an AlGaN structure with flat interfaces due to the effect of total internal reflection. Random surface corrugation occurring on the length scale of the emitting wavelength leads to escape-angle randomization increasing the photon escape probability. ${ }^{8}$ To estimate relative efficiencies of these three mechanisms of the PL enhancement we compare the QW PL intensity in both samples at different temperatures. The integrated PL from the corrugated $\mathrm{QW}$ is $\sim 4$ times stronger at $4.5 \mathrm{~K}$ and $\sim 60$ times stronger at $300 \mathrm{~K}$ as compared to that from the planar QW. Since the effect of the roughness-induced enhancement of the light extraction efficiency should not depend on temperature, we conclude that most of the PL enhancement at elevated temperatures occurs due to combined effects of the suppressed QCSE and the enhanced carrier localization.

\section{CONCLUSIONS}

We have shown that a single $\mathrm{Al}_{\mathrm{x}} \mathrm{Ga}_{1-\mathrm{x}} \mathrm{N} / \mathrm{Al}_{\mathrm{y}} \mathrm{Ga}_{1-\mathrm{y}} \mathrm{N}$ QW grown by plasma-assisted MBE on a c-sapphire substrate with an AlN buffer replicates the morphology of the buffer. Al-rich growth conditions lead to 2D buffer morphology and formation of a planar QW. On the other hand, $\mathrm{N}$-rich growth conditions facilitate realization of 3D buffer morphology and results in a corrugated QW.

This corrugated QW demonstrates significantly improved emission properties. In particular, the QW emission line in this structure is shifted toward higher energies and the emission intensity is enhanced compared to the planar QW by a factor of 4 at $4.5 \mathrm{~K}$ and a factor of 60 at $300 \mathrm{~K}$. One reason behind the improved emitting characteristics is the effect of suppression of the polarization induced electric fields, unambiguously demonstrated by time-resolved PL measurements and supporting variational calculations of the exciton energy and radiative lifetime. Additionally, we spec- ulate that the internal quantum efficiency of the corrugated QW can benefit from stronger carrier localization caused by the enhanced compositional fluctuations in the AlGaN heterostructure with rough 3D morphology. Further work is needed to clarify relative contributions of the two enhancement mechanisms. We also deduced from the temperature dependent measurements that the enhancement of the light extraction efficiency expected for the structure with rough surface hardly exceeds a factor of four in the studied sample.

The observed increase in the PL intensity associated with the enhancement of the internal quantum efficiency makes the structures with corrugated wide $\mathrm{AlGaN}$ QWs very promising for development of high-power deep UV LEDs.

\section{ACKNOWLEDGMENTS}

This work was partly supported by RFBR Grant Nos. 12-02-00856-a, 12-02-00865-a, 13-02-00801-a, and 13-0212231-ofi-m as well as by Program \#24 of Presidium of RAS.

\footnotetext{
${ }^{1}$ A. Khan, K. Balakrishnan, and T. Katona, Nat. Photonics 2, 77 (2008).

${ }^{2}$ H. Hirayama, Y. Tsukada, T. Maeda, and N. Kamata, Appl. Phys. Express 3, 031002 (2010).

${ }^{3}$ Y. Liao, C. Thomodis, C. Kao, and T. D. Moustakas, Appl. Phys. Lett. 98, 081110 (2011).

${ }^{4} \mathrm{R}$. Butté and N. Grandjean, Effects of Polarization in Optoelectronic Quantum Structures, Polarization Effects in Semiconductors, edited by C. Wood and D. Jena (Springer, New York, 2008).

${ }^{5}$ J. E. Northrup, C. L. Chua, Z. Yang, T. Wunderer, M. Kneissl, N. M. Johnson, and T. Kolbe, Appl. Phys. Lett. 100, 021101 (2012).

${ }^{6}$ R. E. Kuokstis, W. H. Sun, C. Q. Chen, J. W. Yang, and M. A. Khan, J. Appl. Phys. 97, 103719 (2005).

${ }^{7}$ K. Kojima, A. A. Yamaguchi, M. Funato, Y. Kawakami, and S. Noda, J. Appl. Phys. 110, 043115 (2011).

${ }^{8}$ J. S. Cabalu, C. Thomidis, T. D. Moustakas, S. Riyopoulus, L. Zhou, and D. J. Smith, J. Appl. Phys. 99, 064904 (2006).

${ }^{9}$ V. N. Jmerik, T. V. Shubina, A. M. Mizerov, K. G. Belyaev, A. V. Sakharov, M. V. Zamoryanskaya, A. A. Sitnikova, V. Yu. Davydov, P. S. Kop'ev, E. V. Lutsenko, N. V. Rzheutskii, A. V. Danilchyk, G. P. Yablonskii, and S. V. Ivanov, J. Cryst. Growth 311, 2080 (2009).

${ }^{10}$ V. N. Jmerik, A. M. Mizerov, A. A. Sitnikova, P. S. Kop'ev, S. V. Ivanov, E. V. Lutsenko, N. P. Tarasuk, N. V. Rzheutskii, and G. P. Yablonskii, Appl. Phys. Lett. 96, 141112 (2010).

${ }^{11}$ P. Cantu, F. Wu, P. Waltereit, S. Keller, A. E. Romanov, U. K. Mishra, S. P. DenBaars, and J. S. Speck, Appl. Phys. Lett. 83, 674 (2003).

${ }^{12}$ Y. Shinozuka and M. Matsuura, Phys. Rev. B 28, 4878 (1983).

${ }^{13}$ M. Grundmann and D. Bimberg, Phys. Rev. B 38, 13486 (1988).

${ }^{14}$ I. Vurgaftman and J. R. Meyer, J. Appl. Phys. 94, 3675 (2003).

${ }^{15}$ H. Murotani, T. Kuronaka, Y. Yamada, T. Taguchi, N. Okada, and H. Amano, J. Appl. Phys. 105, 083533 (2009).

${ }^{16}$ B. Monemar, P. P. Paskov, J. P. Bergman, A. A. Toropov, and T. V. Shubina, Phys. Status Solidi B 244, 1759 (2007).

${ }^{17}$ K. P. O’Donnel, P. J. Parbrook, F. Yang, and C. Trager-Cowan, Physica B 191, 45 (1993).

${ }^{18}$ D. S. Citrin, Phys. Rev. B 47, 3832 (1993).

${ }^{19}$ M. Sugawara, Phys. Rev. B 51, 10743 (1995).

${ }^{20}$ M. Gao, S. T. Bradley, Y. Cao, D. Jena, Y. Lin, S. A. Ringel, J. Hwang, W. J. Schaff, and L. J. Brillson, J. Appl. Phys. 100, 103512 (2006).

${ }^{21}$ A. V. Sampath, G. A. Garrett, C. J. Collins, W. L. Sarney, E. D. Readinger, P. G. Newman, H. Shen, and M. Wraback, J. Electron. Mater. 35, 641 (2006).

${ }^{22}$ V. Fellmann, P. Jaffrennou, D. S. Giao, B. Gayral, K. Lorenz, E. Alves, and B. Daudin, Jpn. J. Appl. Phys. 50, 031001 (2011).
} 